opportunities for occupational and environmental particulate matter measurement system.

Acknowledgement This work was supported by Gachon University research fund of 2018(GCU-2018-5259) and the National Research Foundation of Korea (NRF) grant funded by the Korea government (Ministry of Science, ICT) (No. NRF-2017R1C1B1002717).

\section{P.1.15 MERCURY HAZARDS AND HOW THE COMMUNITY RESPONSES IN DEVELOPING COUNTRY}

Shambhu Joshi. Far Western Community Hospital, Kailali, Nepal

\subsection{6/OEM-2019-EPI.218}

Introduction Heavy metal like mercury was used directly or indirectly since long ago before it was stopped around the world. Since, the value of the metal and its availability in different use in medical appliances is still one of the burdens in developing country like Nepal. The main uses of mercury are in dental amalgam, sphygmomanometers, and thermometers. The mercury once released into the environment can remain for a longer period. Both acute and chronic poisoning can be caused by it. Half of the mercury found in the atmosphere is human generated and health care contributes the substantial part to it. WHO and UNEP issued new guideline for health care sector to become mercury free.

Aim and methods To find out the Knowledge, attitude and practice due to hazards of mercury contact among paramedics and patients.

Results and conclusion 938 paramedics and 890 patients were asked indepth structured questions about mercury hazards and update knowledge. Overall $18 \%$ of the paramedics have no knowledge of mercuryfree with respect to $69 \%$ of the patients. Around $49 \%$ of paramedics have broken mercury thermometer in their career and contact with skin. However, $1.2 \%$ patient only have a similar history in their life time. $4 \%$ of the contamination with mercury having SKIN problems. $32 \%$ of the patients still have mercury thermometer and sphygmomanometer in their home with respect of $0.6 \%$ of paramedics. The above study showed that Healthcare worker has more knowledge of Mercury hazards than patients groups. However, paramedics have broken the mercury instruments than patients party. Similarly, Paramedics have Mercury free device at home than patients groups. It means awareness, literate people having more knowledge towards its practice making mercury free society in a resource poor country like Nepal.

\section{P.1.16 PHYSICAL HEALTH OF TRANSITIONED ADF AND REGULAR ADF MEMBERS IN 2015: FINDINGS FROM THE TRANSITION AND WELLBEING RESEARCH PROGRAMME}

\footnotetext{
${ }^{1}$ Helen Kelsall ${ }^{*},{ }^{2}$ Miranda Van Hooff, ${ }^{2}$ Ellie Lawrence-Wood, ${ }^{2}$ Alexander McFarlane, ${ }^{3}$ Stephanie Hodson, ${ }^{4}$ Nicole Sadler, ${ }^{5,6}$ Helen Benassi, ${ }^{2}$ Craig Hansen, ${ }^{1}$ Malcolm Sim. ${ }^{1}$ School of Public Health and Preventive Medicine, Monash University, Melbourne, Australia; ${ }^{2}$ Centre for Traumatic Stress Studies, University of Adelaide, Adelaide, Australia; ${ }^{3}$ Veterans and Veterans Families Counselling Service, Department of Veterans' Affairs, Melbourne, Australia; ${ }^{4}$ Phoenix Australia Centre for Posttraumatic Mental Health, University of Melbourne, Melbourne, Australia; ${ }^{5}$ Mental Health, Rehabilitation and Psychology Branch, Department of Defence, Canberra, Australia; ${ }^{6}$ Australian National University, Canberra, Australia
}

10.1136/OEM-2019-EPI.219
Introduction Military service can involve exposure to physical and psychological stressors. There has been little systematic research into the health and wellbeing of military personnel after they leave the services.

Methods 4326 Transitioned ADF (transitioned from regular Australian Defence Force service between Jan 2010-Dec 2014) (18\% response) and 8480 Regular 2015 ADF (42\%) completed a questionnaire including symptoms, doctor-diagnosed medical conditions, respiratory health, injuries, pain, sleep problems, lifestyle factors, self-perceived health and quality of life and health service use.

Results Transitioned ADF reported a higher mean number of symptoms (16.4 vs 11.8), similar mean number of medical conditions (1.9 vs 1.5), were more likely to report some medical conditions (a circulatory, musculoskeletal/connective tissue or nervous system condition, high blood pressure, chronic low back pain, and hearing loss), a slightly higher mean number of service-related injury types (1.11 vs 0.96 ), and poorer selfperceived health and quality of life compared to 2015 Regular ADF. Service-related injuries were more likely to have been sustained during training than on deployment in both groups. The majority of Transitioned ADF and 2015 Regular ADF reported experiencing some pain intensity and disability.

In Transitioned ADF, poorer physical health outcomes overall were reported in Department of Veterans' Affairs (DVA) clients compared with non-DVA clients, in Ex-Serving compared with Active Reservists or Inactive Reservists, and in those who had been medically discharged compared with those discharged for other reasons.

Conclusion This was one of the first studies internationally to investigate a comprehensive range of physical health indicators in recently transitioned military personnel. Overall Transitioned ADF were more likely to report poorer physical health across domains, some subgroups appeared particularly at risk. Findings in DVA clients were consistent with DVA being the conduit for care in veterans who have a service-related injury or mental health condition.

\section{P.1.17 WORKING TIME, SMOKING, AND CAFFEINE INTAKE BY WORKING TYPE OF TAXI DRIVERS IN KOREA}

Hyoung-ryoul Kim*. Department of Occupational and Environmental Medicine, College of Medicine, The Catholic University of Korea, Seoul, South Korea

\subsection{6/OEM-2019-EPI.220}

Taxi drivers in Korea are known to work long hours and receive low wages. In addition, there are various forms of working, so that they can work 12 hours a day for 2 shifts, work only at night or day time, and drive a car whole day alone (14-15 hours a day). We surveyed their specific hours of work, and smoking and caffeine consumption. In this study, 11 business sites were selected for taxi companies in Seoul considering region and workplace size, and survey was conducted for all taxi drivers belonging to the relevant business sites. The questionnaire consisted of demographic characteristics, working hours and working conditions, violence experience, emotional labor status, physical and mental health status, sleep health, traffic accidents and traffic violation experience. A total of 698 respondents (39.6\%) answered the questionnaire. $76.2 \%$ were working $25-26$ days a month. $49.2 \%$ of the workers worked more than 60 hours per week and less than 70 hours per week. $52.8 \%$ of drivers were current smokers, and $65.9 \%$ of those who work fixed night shift were current 